\title{
Commentary on Pragmatic Case Studies and Evidence-Based Treatment: Research and Clinical Applications of a Computerized Outcomes Management System
}

\section{Empirical Evaluation of the Utility and Effectiveness of Clinically Flexible Application in Evidence-Based Treatments}

\author{
TIMOTHY R. STICKLE ${ }^{\mathrm{a}, \mathrm{b}}$
}

\footnotetext{
${ }^{\mathrm{a}}$ Department of Psychology, University of Vermont, Burlington

${ }^{\mathrm{b}}$ Correspondence concerning this article should be addressed to Timothy R. Stickle, Department of Psychology, John Dewey Hall, University of Vermont, Burlington, VT 05405 Email: tstickle@uvm.edu
}

\begin{abstract}
A common criticism of the adoption and use of empirically supported treatments is their perceived lack of applicability to complex individual cases. For example, some individual clients present with multiple problems, comorbid conditions, or respond poorly to the best available psychological treatment. An ongoing dilemma for scientifically-minded clinicians in such situations is whether and how best to proceed with deviations from manualized treatments. Conscientious clinical scientists may be reluctant to deviate from protocol without some means of assessing whether outcome will be better or worse than proceeding with the protocol. Some clinicians may abandon the protocol and the principles on which it is based, and proceed as guided by clinical judgments based on recollections of similar cases and roadblocks. How does the conscientious clinical scientist evaluate whether deviations from treatment protocol enhance or degrade outcome? I will discuss the relative merits and possibilities of the Polaris-MH-datainformed approach provided by Grissom and Lyons (2006), with attention to (a) its potential for providing evidence-based feedback to clinician and client, and (b) how it might be particularly useful in the context of empirically-supported treatments. I will also propose principles for how clinicians can use evidence from relevant literature to inform and evaluate the utility of deviations from protocol.
\end{abstract}

Key words: empirically supported treatments (ESTs); complex individual cases; comorbidity; manualized treatment; therapy outcome

\section{INTRODUCTION}

Although empirically-supported treatments (ESTs) have notable evidence of efficacy (Chambless, Baker, Baucom, Beutler, et al., 1998), they are not yet widely used in daily practice 
and remain controversial among psychologists. Additionally, many clinicians criticize ESTs for their perceived lack of applicability to complex cases (see e.g., Persons and Silberschatz, 1998). Even for clinicians who regularly employ ESTs in practice, many of these treatments have unsatisfactory outcomes with some individuals. Among common criticisms are questions of the applicability of many ESTs to individuals with multiple problems, comorbid diagnoses, and "non-responders" to the treatment. In practice and training settings, these are important questions that appear to lead to a variety of attempted solutions.

One solution appears to be abandoning manualized protocols, typically by employing an eclectic approach. An eclectic approach generally augments the initial treatment approach by drawing on one other or multiple other theories of problems and change. Systematic study of such approaches is sparse, although such approaches appear to be common in practice (Norcross and Goldfried, 1992). Although an eclectic approach to treatment augmentation has some appeal based on its perceived relevance to immediate clinical phenomena and its potential to involve clinicians in refining treatments (Goldfried and Wolfe, 1998), it remains unclear how best to piece together components of therapies, processes, and strategies.

Another solution sometimes employed is to target the most distressing problem and proceed with other problems as the initial one diminishes or is resolved. Although such an approach can be effective, it is often unclear how to determine which problem is primary and how changes in one set of concerns and symptoms may be impeded by other problems. In the latter case, the apparently primary problem may be affected by the apparently secondary problem, and treatment progress may subsequently be slowed more by the problem left unaddressed than by lack of progress with the target problem. Although functional analysis and other approaches can and should be used in such cases, this in itself may be a deviation from the usual protocol and leave the same questions about how to evaluate effectiveness of such adaptation relative to adherence to protocol.

I am a strong advocate of empirically supported approaches to treatment, or what Grissom and Lyons (2006), after Howard, Moras, Brill, \& Martinovich (1996), term the "treatment-focused research" model. The model, in my view, receives undue criticism about lack of flexibility when such flexibility not only is possible, but is inherently part of many current treatment manuals. There is nothing about manualized and empirically supported treatments that requires the clinician to behave incompetently. That is, rigid adherence to protocol when it clearly is ineffective, when clients are in crisis, and so forth, is a limitation of application of the treatment by the clinician, not of the treatment itself.

Nevertheless, an important and real dilemma for conscientious, scientifically-minded clinicians is how to proceed in such cases. How one makes informed, evidence-based decisions and evaluates them is among the most important questions facing practicing clinical scientists and training programs. Scientific creativity and application of empirically-supported principles of change and thoughtful use of extant experimental literature are possible approaches to flexible and informed application of ESTs. 
Grissom and Lyons' (2006) description of Polaris-MH, a computer-based, outcomes management system, suggests that it has promise as a valuable tool by which clinicians can evaluate the effectiveness and utility of flexible application of ESTs, and of empiricallyinformed deviations from good treatments that are less than effective in particular cases. In the remainder of this paper, I attempt to outline how Polaris-MH could be used to gather and evaluate evidence across multiple cases for which ESTs failed to achieve expected results. Additionally, I suggest principles for guiding intervention in this context and for how the data from Polaris-MH might be used to inform and refine flexible application of ESTs.

\section{POLARIS-MH AND PRACTICE-INFORMED RESEARCH}

The Grissom and Lyons (2006) description of Polaris-MH suggests its promise for application to multiple cases, as it takes into account available data on dose-response, treatment phase, and on expected treatment response. Polaris-MH can make use of a wide variety of measures, large numbers of cases, and contemporary data analytic approaches, such as hierarchical linear modeling (HLM) and individual growth curve analysis, allowing tracking of both individual cases and groups of cases that may be nested within treatment site, clinician, or other organizational structure that might lead to higher correlations within that grouping. These nesting units, which are referred to as Level 2 units in HLM, can lead to bias in analyses unless taken into account statistically as is done in HLM. For example, although we usually consider individual clinicians separately, clinicians within the same organization or treatment site may have more similar outcomes to each other than with clinicians from other locations. These higher, within-site correlations may bias comparisons of specific treatment approaches within and between sites. HLM and related data analytic approaches account for this potential bias statistically by expressing relationships within each level and how variables at one level, such as organizational policy and/or clinical supervision, influence relations at another level, such as treatment delivery, length of treatment, and/or treatment fidelity (Raudenbush \& Bryk, 2002). As a result of these capabilities, contemporary data analytic approaches provide improved assessment of clinician effects, treatment effects, and site (organizational) effects on individual and grouped outcomes for individual clients.

Use of growth curve modeling represents a potentially important contribution to tests of patient characteristics and treatment response. Because of the capability of growth curve analyses to estimate a separate change trajectory for each individual in the analysis (i.e., a random effects model), such analyses can estimate a unique starting point (intercept) and trajectory (slope) across treatment for each individual case. That is, rather than estimating only a single intercept and slope for all cases as in a fixed effects (e.g., multiple regression, standard ANOVA) analysis, each individual in the sample can be modeled on a true trajectory, rather than estimating only each individual's similarity to the average trajectory. Similarly, groups of cases with common characteristics can be identified by similar starting points and/or trajectories. Additionally, the effects of various starting points (e.g., starting points differentiated by severity of presenting problems) on the trajectory of change (or lack of change) can be highly informative 
about ways in which pre-treatment characteristics may affect progress across the course of treatment.

For example, such characteristics could easily be separated by those that are changeable and might be treatment targets and those that are not changeable. The relationships of each of these sets of characteristics to change trajectories should inform both expected treatment response curves and treatment selection and planning. As a result, this technology looks to have important potential for informing research questions within clinical trials as well as for informing the clinician and client in individual cases.

\section{POTENTIAL DIFFICULTIES WITH USE OF POLARIS-MH}

Expected treatment response (ETR) forms the baseline slope estimate for each individual or set of individuals. The success of this technology in informing ESTs and ongoing practice rests heavily on the validity of the ETR estimates. This validity question is of greatest concern to me at this stage of development of Polaris-MH. It is not yet clear how closely such response curves correspond to what should, in fact, be expected in individual cases for several reasons.

First, although Grissom and Lyons (2006) note high correlations between measures used by Polaris-MH and measures used in comparative studies of non-clinical populations, the extent to which these estimates form boundaries around adaptive and maladaptive functioning is uncertain. This uncertainty is because the Polaris-MH scales have not been used in non-patient populations. Despite high correlations of those scales with scales used in other studies, norms and construct validity are not yet established. A more formal calibration of the relations among Polaris-MH measures and commonly used measures would provide such evidence (Sechrest, McKnight, \& McKnight, 1996). The correlations among, for example, the Polaris-MH Subjective Well-Being scale and the General Well-Being Scale (GWB; Dupuy, 1977) and the Positive and Negative Affect Scale (PANAS; Watson \& Tellegen, 1985) form the basis of inferences about clinical significance (i.e., the reliable change index [RCI], developed by Jacobson and Truax (1991). Although the correlation between the Mental Health Index from other studies and the Subjective Well Being scale used in Polaris-MH is high and the scales similar, the error around estimates of clinical significance is likely fairly large. For example, if the correlation between the two measures is .80, they share about $64 \%$ common variance. Because the RCI is based on a cutoff score, the accuracy of that score is diminished by inferring it is the same for two measures that correlate less than unity. The extent to which the estimates deviate from 1.0 will vary, greatly in some cases, for different problems and types of individuals. Because there are not established norms by which to be confident that an individual is in the functional vs. impaired range, more evidence should be generated before accepting the authors' assertion that collection of data from non-patients is unnecessary.

Some important published data indicate poor prediction of treatment outcome based on patient characteristics, for example, Project MATCH (Project MATCH Research Group, 1997). This literature suggests the need for more data on the use of Polaris-MH for cumulative case 
studies of flexible or individualized application of treatment protocols based on such characteristics before more confidence can be put in it, particularly because of the lack of norms for cut-offs between clinical and non-clinical individuals on the outcome measures. It is simply not known what the functional vs. impaired ranges are yet.

Lastly, previous comparisons of clinically-flexible vs. research-structured treatment protocols indicate that differences may also be evident in durability of effects (Jacobson, Dobson, Truax, Addis, et al., 1996). Therefore, it is important to examine ETR curves both during treatment and for sufficient follow-up periods. Clinical trials increasingly examine longer follow-up results, and comparisons with such outcomes should become increasingly feasible.

The foregoing concerns should not detract from what looks to be an interesting and exciting tool for continued refinement of empirically supported treatments in daily practice. Additionally, ongoing data and feedback should help to promote both refinements in practice and in research protocols. That is, if particular adaptations of treatment protocols provide consistent improvement in outcome, research designs for randomized trials can be informed by those results from clinical practice.

\section{THE USE OF POLARIS-MH}

The literature on clinical judgment for guiding treatment decisions suggests that clinical judgment is unlikely to provide consistent, predictable guidance for deviation from evidencebased treatment protocols (e.g., Dawes, Faust, and Meehl, 1989; Garb 1997). In keeping with an evidence-based approach to treatment, there are likely several possible approaches to using treatment response curves and patient characteristics as the basis for flexible application of ESTs.

One such approach that I propose here has two primary guiding principles.

Understanding the basic psychopathology under treatment and how the treatment is supposed to work to alter it are foundational. Although some treatments may work for reasons we don't understand, those treatments probably cannot be adapted in thoughtful and predictable ways in response to case information. In the situation where we have some idea of the basic processes of psychopathology and treatment mechanisms, however, such modifications might be fruitful.

For example, exposure treatment for phobia is based primarily on principles of learning and conditioning. The evidence for efficacy of exposure for the treatment of specific phobia is strong (Chambless et al., 1998). In some cases, however, additional problems and mediating mechanisms may require targeting. The experimental psychopathology literature provides empirical evidence about such potential mechanisms. For example, it appears relatively common among individuals experiencing some specific phobias (e.g., phobia of receiving an injection for medical reasons) to experience a strong disgust reaction to anticipation of the feared stimulus. Additionally, this literature also suggests that targeting disgust may provide mechanisms that facilitate successful exposure (Tolin, Sawchuk, \& Lee, 1999; Sawchuk et al., 2002). Thus, when the feared object or situation (e.g., a needle) elicits disgust, including desensitization to the 
disgust response through exposures to progressively more disgusting stimuli appears to facilitate successful exposure to the feared situation targeted (e.g., receiving an injection for medical reasons).

The preceding, relatively simple, example is provided to illustrate how clinicians might query about potential roadblocks and change mechanisms in cases of non-response. That is, acquaintance with more basic research on psychopathology can importantly alert clinicians to inquire about the presence of disgust reactions, since clients on their own may not report such a response, and exposure protocols do not yet incorporate this mechanism.

There are numerous similar examples related to attributional processes within depression, anxiety, and aggression; to physiological responses within anxiety pathology; and so forth. Using the experimental psychopathology literature to inform targets for change represents one avenue of applying psychological science in psychotherapy.

Lastly, by understanding how treatments are intended to achieve results via researchderived and research-supported change mechanisms within different types of psychopathology, clinicians can think creatively about how to employ those principles in individual cases. In a related vein, an evidence-based approach can and probably should examine application of the treatment principles in different orders and in different ways. Within all of these considerations, Polaris-MH shows promise as a tool to gather ongoing data and provide feedback to the application of clinical science in daily practice.

\section{REFERENCES}

Chambless, D.L., Baker, M.J., Baucom, D.H., Beutler, L.E., Calhoun, D.S., Crits-Cristoph, P., Daiuto, A., DeRubeis, R., Detweiler, J., Haaga, D.A.F., Johnson, S.B., McCurry, S., Mueser, K.T., Pope, K.S., Sanderson, W.C., Shoham, V., Stickle, T., Williams, D.A., \& Woody, S.R. (1998). Update on empirically validated therapies, II. The Clinical Psychologist, 51(1), 3-16.

Dawes, R.M., Faust, D. \& Meehl, P.E. (1989). Clinical versus actuarial judgment. Science, 243, 1668-1674.

Dupuy, H. J. (1977). A current validational study of the NCHS general well-being schedule. (DHEW Publication No. HRA 78-1347). Hyattsville, MD: National Center for Health Statistics, US Department of Health, Education, and Welfare.

Garb, H. (1998). Studying the clinician: Judgment research and psychological assessment. Washington, DC: American Psychological Association.

Goldfried, M.R. \& Wolfe, B.E. (1998). Toward a more clinically valid approach to therapy research. Journal of Consulting and Clinical Psychology, 66(1), 143-150.

Grissom, G. \& Lyons, J. (2006). Pragmatic case studies and evidence-based treatment: Research and clinical applications of a computerized outcomes management system. Pragmatic Case Studies in Psychotherapy [Online], Vol. 2(3), Article 1. Available: http://hdl.rutgers.edu/1782.1/pcsp_journal. 
Howard, K.I., Moras, K., Brill, P.L, \& Martinovich, Z. (1996). Evaluation of psychotherapy: Efficacy, effectiveness, and patient progress. American Psychologist. 51, 1059-1064.

Jacobson, N.S, Dobson, K.S., Truax, P.A., Addis, M.E., Koerner, K., Gollan, J.K., Gortner, E, \& Prince, S.E. (1996). A component analysis of cognitive-behavioral treatment for depression. Journal of Consulting and Clinical Psychology, 64, 295-304

Jacobson, N.S. \& Truax, P. (1991). Clinical significance: A statistical approach to defining meaningful change in psychotherapy research. Journal of Consulting and Clinical Psychology, 59, 12-19.

Norcross, J.C. \& Goldfried, M.R. (Eds.) (1992). Handbook of psychotherapy integration. New York: Basic Books.

Project Match Research Group. (1997). Matching alcoholism treatments to client heterogeneity: Project MATCH Posttreatment drinking outcomes. Journal of Studies on Alcohol, 58(1), 7-29.

Persons, J.B. \& Silberschatz, G. (1998). Are results of randomized controlled trials useful to psychotherapists? Journal of Consulting and Clinical Psychology, 66(1), 126-135.

Raudenbush, S.W. and Bryk, A.S. (2002). Hierarchical linear models: Applications and data analysis methods, $2^{\text {nd }}$ Ed., Thousand Oaks, CA: Sage.

Sawchuk, C.N., Lohr, J.M., Westendorf, D.H., Meunier, S.A., \& Tolin, D.F. (2002). Emotional responding to fearful and disgusting stimuli in specific phobics. Behaviour Research and Therapy, 40(9), 1031-1046.

Sechrest L, McKnight PE, McKnight KM. (1996). Calibration of measures for psychotherapy outcome studies. American Psychologist, 51, 1065-1071

Tolin, D.F., Sawchuk, C.N., Lee, T.C. (1999). The role of disgust in blood-injection-injury phobia. the Behavior Therapist, 22(5), 96-99.

Watson, D., \& Tellegen, A. (1985). Toward a consensual structure of mood. Psychological Bulletin, 98, 219-235. 